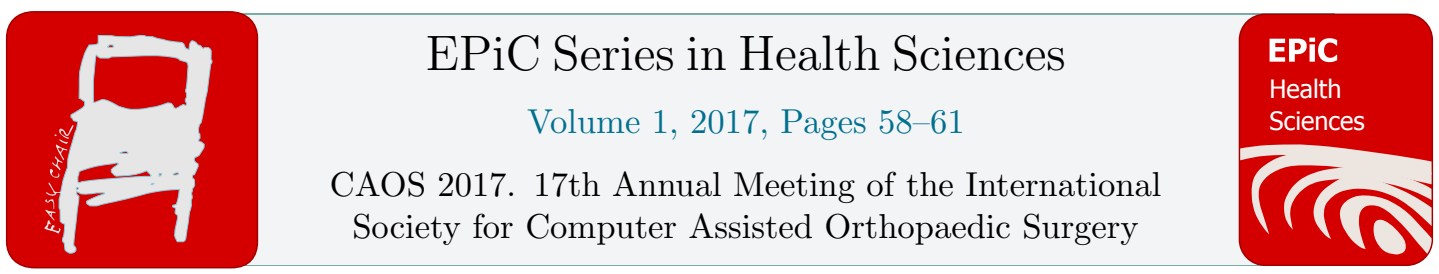

\title{
Smart device based application for rod determination in minimal invasive spine surgery
}

\author{
Andreas Alk ${ }^{1,2}$, Tobias Martin ${ }^{1,2}$ and Josef Kozak ${ }^{1,2}$ \\ ${ }^{1}$ A esculap AG, Tuttlingen, 78532, Germany \\ ${ }^{2}$ AGH University of Science and Technology, Krakow, 30-059, Poland \\ andreas.alk@aesculap.de, tobias.martin@aesculap.de, \\ josef. kozak@aesculap.de
}

\begin{abstract}
In orthopaedic spine surgery pedicle screw systems are used for stabilisation of the spine after injuries or disorders. With an percutaneous operation method surgeons are faced with huge challenges compared to an open surgery, but it`s less traumatic and the patient benefits with a faster rehabilitation and less traumatic injuries. The screw positions and the required rod dimensions for the stabilizing connection between the screws are hard to define without an open view on the operating field. Because of these facts a new smart device based system for rod shape determination was invented. Therefore, an application was developed, which integrates a localizer module to get the position data of the pedicle screws, with help of rigid bodies placed on top of the pedicle screws down-tubes. An algorithm was developed to choose the best fitting rod to connect the pedicle screws with help of calculating the rod length and the rod radius. The system was tested in a test scenario where four pedicle screws were drilled into a wooden plate. The positions of the screws were adjusted to fit a curved and a straight rod. In the test scenario the application chose always the rod correctly.
\end{abstract}

\section{Introduction}

Minimally invasive surgeries are getting more and more popular in the field of medicine. The benefit for the patients is huge. It is less traumatic, that is why the patient profits from a shorter hospital stay and a faster recovery. In orthopedics spine surgery the gold standard for fixation of the spine are pedicle screw systems. Pedicle screws are used in several indications for example for spondy lolisthesis and fractures of the vertebrae to stabilize and correct the vertebrae dis placements of the spine, or for correction of deformities like scoliosis. Conventional surgical procedures for posterior spinal fusion require large incisions, extensive injuring and retraction of the paraspinal musculature with significant postoperative pain and long recovery times (Harris 2008). Due to the fact that it is less traumatic than the conventional method, the minimally invasive, percutaneous surgery 
methods (MIS) becomes increasingly popular in the field of lumbar pedicle screw procedure. MIS relies also greatly on the surgeon's overall skills as well as the patient selection - not each indication is the fit for MIS. The benefits of MIS are huge, but the operating technique confronts the surgeon and his team with serious challenges and a higher radiation load exposure (Mroz 2011). During a surgery, a fitting rod which fits the pedicle screws must be determined, without a direct view on the operating field. It is possible to get the pedicle screws' positions with a longitudinal X-Ray Image to see their position in a 2D view on the sagittal plane. To reduce the radiation load of this additional image, mechanical systems are invented to define the rod length and the rod shape. In this paper a new digital method, for determining a fitting rod with a mobile medical localizer that records the position of the reflective markers positioned on the minimal invasive down tubes, is presented (Daniol 2015).

\section{Materials and Methods}

The hardware of the rod determination system consis ts of Aesuclap's S4 Element ${ }^{\circledR}$ pedicle screw system for MIS TLIF surgery with special adapters for two different Rigid Bodies with retro reflective markers and a medical mobile localizer with a custom developed application. The medical mobile localizer detects the retroreflective markers of the Rigid Bodies and calculates the position of the pedicle screw heads, which the Rigid Bodies are attached to like presented in Figure 1. For the measurement of the positions one pedicle screw is fixed and serves as a reference screw. During the whole recording a non-movable Rigid Body is positioned on the top of the reference screw's downtube. Further, three remaining pedicle screws are localized with the mobile Rigid Body attached to them. During the recordings the mobile Rigid Body is moved from the nearest to the furthest positioned screw with regard to the fixed screw.

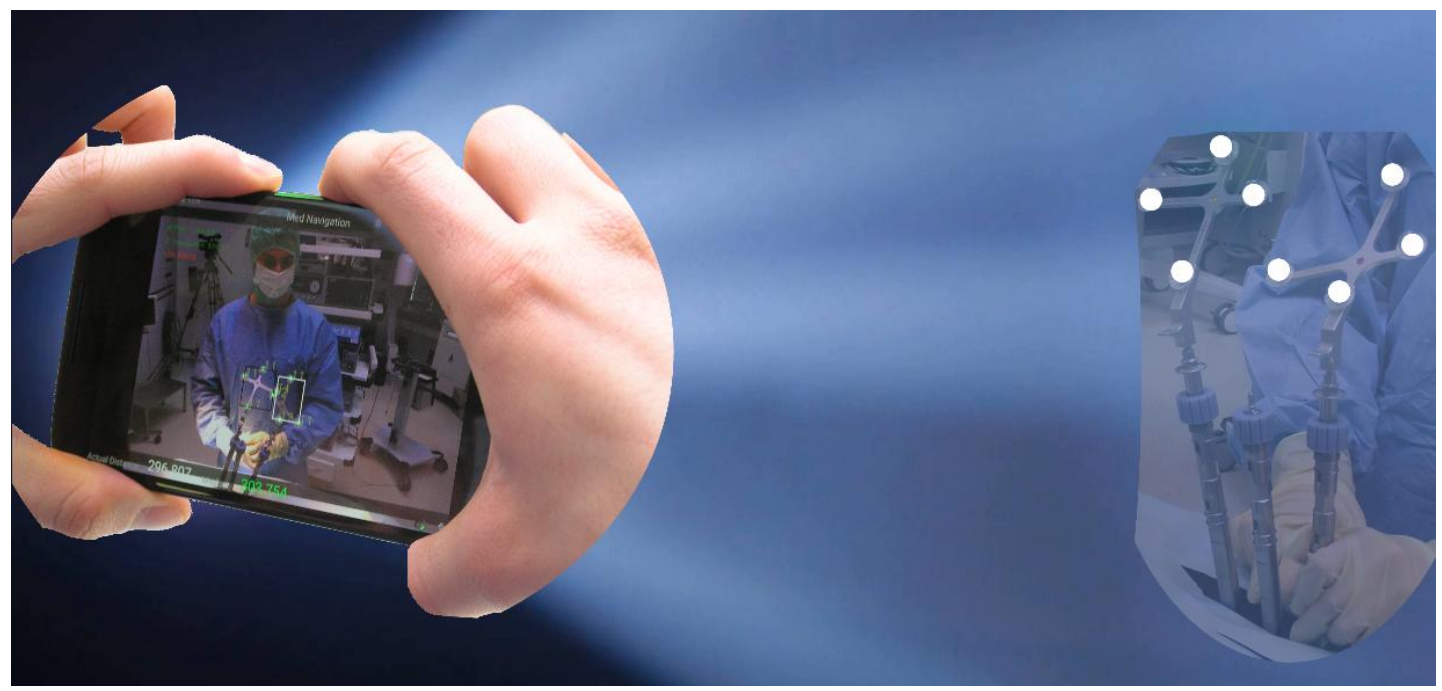

Figure 1: System for rod determination

Before the mobile Rigid Body is moved to the next screw a surgery assistant presses the record button in the application. After finishing the recordings, the algorithm calculates the rod length and the rod radius and chooses the best fitting rod to connect the pedicle screws. The results are displayed graphically on a result screen, as demonstrated in Figure 2.

In the test scenario four pedicle screws were drilled into a wooden plate. The positions of the screws were adjusted to fit a curved and a straight rod. For each rod ten measurements were 
performed and the correctness of the algorithm for selecting the best fitting rod was tested. The calculated rod length and rod radius were measured as well.

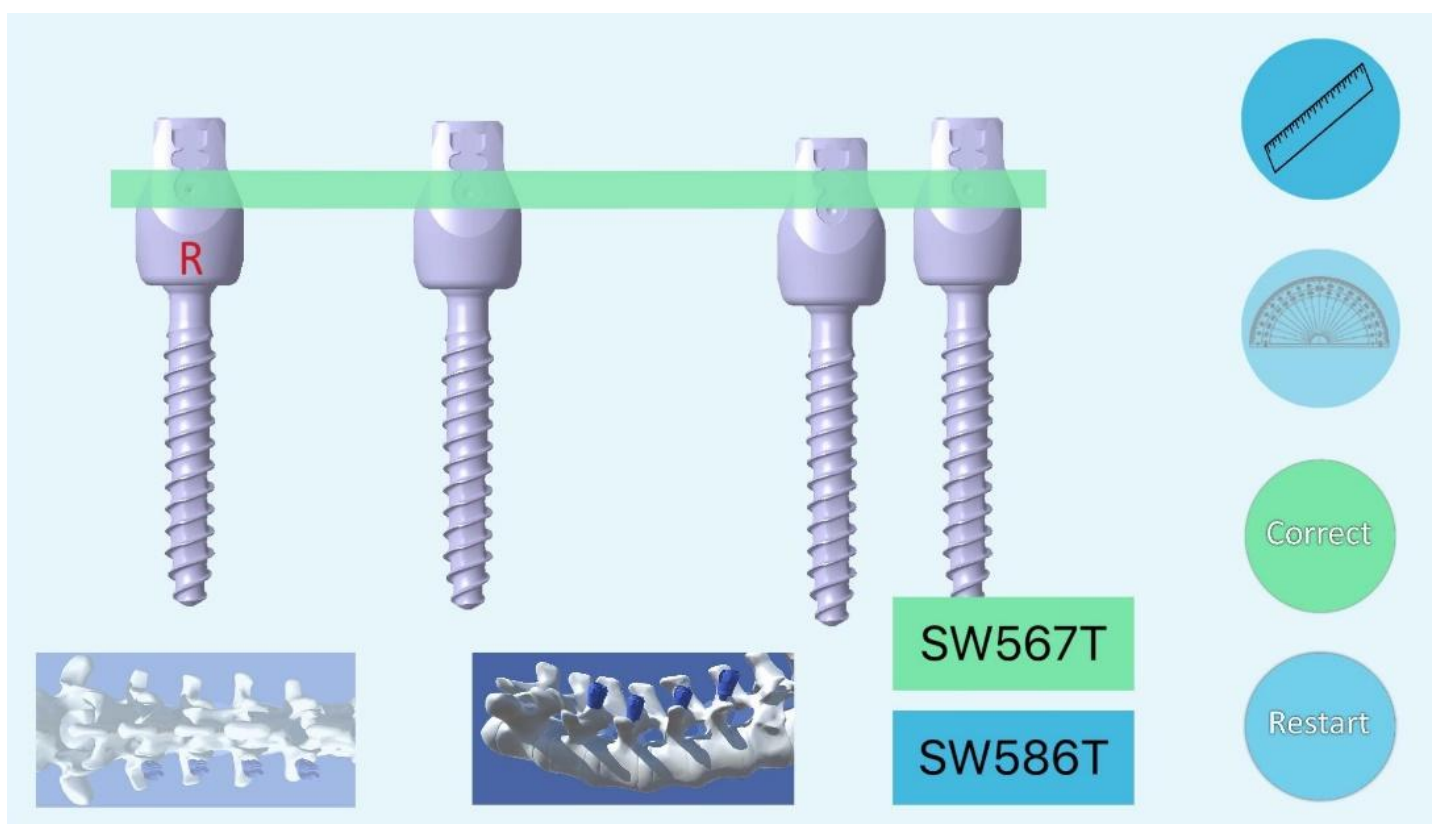

Figure 2: System for rod determination

\section{Results}

The application always chose the correct rod for the straight and curved pedicle screw positions in the test scenario. The mean value of the curved rod length was $119.48 \mathrm{~mm}$ with a standard deviation of $\pm 0.73 \mathrm{~mm}$. The mean value of the curved rod radius was $80.73 \mathrm{~mm}$ with a standard deviation of $\pm 14.19 \mathrm{~mm}$. The mean value of the straight rod length was $118.24 \mathrm{~mm}$ with a standard deviation of $\pm 0.79 \mathrm{~mm}$. The mean value of the curved rod radius was $307.7 \mathrm{~mm}$ with a standard deviation of $\pm 19.65 \mathrm{~mm}$.

\section{Discussion}

The results of the test scenario show that a smart device based tracking system is able to choose the shape of a rod with tracking the position of the pedicle screw heads correctly. The system is easy to use and no additional radiation is needed. The system is able to solve the huge challenge of rod determination in MIS described by Mobbs et al. (Mobbs 2011). The application displays the location of the pedicle screws relative to each other in different 2D views. Additionally, the sagittal view offers the opportunity to correct the depth position of not accurately fitting pedicle screws for the determined rod. Paik et al. made biomechanical tests of reduction maneuvers of the rod and found out that this maneuverhad a big influence in the peak pullout strength which could lead to the failure of a pedicle screw system (Paik 2013). With the presented system it is possible to correct the depth 
position of the not fitting pedicle screws before the rod is inserted. Such a solution may lead to avoiding the need for additional reduction maneuvers and decreasing the risk of the pedicle screw system failure.

\section{References}

Daniol M, M. T. (2015). Development, calibration, validation and comparison of mobile medical localizers based on smartphones Apple iPhone 5s and Samsung Galaxy S4. Przeglad Elektrotechniczny, pp. 53-56.

Harris EB, M. P. (2008). Percutaneous techniques for minimally invasive posterior lumbar fusion. Joumal of Neurosurgery.

Mobbs RJ, S. P. (2011). Technique, challenges and indications for percutaneous pedicle screw fixation. Joumal of Clinical Neuroscience, pp. 741-749.

Mroz TE, A. K. (2011). Radiation exposure to the surgeon during percutaneous pedicle screw placement. Clinical Spine Surgery, pp. 264-267.

Paik H, K. D. (2011). The biomechanical consequences of rod reduction on pedicle screws: should it be avoided? The Spine Journal, pp. 1617-1626. 